\title{
Guiding of a Slow Positron Beam with a Glass Capillary
}

\author{
N. Oshima ${ }^{1, a}$, Y. Iwai ${ }^{2}$, T. M. Kojima ${ }^{2}$, T. Ikeda ${ }^{2, b}$, Y. Kanazawa ${ }^{3}$, M. Hoshino ${ }^{3}$, \\ R. Suzuki ${ }^{1, c}$, and Y. Yamazaki ${ }^{2}$ \\ ${ }^{1}$ National Institute of Advanced Industrial Science and Technology (AIST), AIST Central-2, 1-1-1 \\ Umezono, Tsukuba, Ibaraki 305-8568, Japan \\ ${ }^{2}$ Atomic Physics Laboratory, RIKEN, 2-1-1 Hirosawa, Wako, Saitama 351-0198, Japan \\ ${ }^{3}$ Department of Physics, Sophia University, 7-1 Kioicho, Chiyoda, Tokyo 102-8554, Japan \\ anagayasu-oshima@aist.go.jp, ${ }^{b}$ tokihiro@riken.jp, ${ }^{c}$ r-suzuki@aist.go.jp
}

Keywords: positron microbeam, glass capillary, beam guiding effect, beam focusing effect.

\begin{abstract}
Slow positron beam was injected into a non-tapered glass capillary which was tilted angle of $\theta$ from the beam axis by a movable stage. Beam profiles of the positrons transported through the capillary were observed with a phosphor screen combined with micro channel plates as a function of $\theta$. Some fraction of positrons was deflected with the tilting angle of the capillary.
\end{abstract}

\section{Introduction}

Typical diameter of a slow positron beam is more than a few mm with an intensity of $10^{5}-10^{7} \mathrm{e}^{+} / \mathrm{s}$. Then, an effective focusing system is required in order to investigate a sample which is smaller than a few mm using a slow positron beam within reasonable measurement time. Focusing system of slow positron beams have been developed by several group, in which electrostatic or magnetic lenses are used [1].

Focusing and guiding techniques of the ion [2, 3] and muon [4] beams with glass capillaries/tubes have been studied in recent years. This focusing technique has some unique features. For example, the diameter of the focused beam at the exit of the capillary is defined clearly by the inner diameter of the capillary exit and dose not depend on the beam properties such as energy, intensity and divergence. A thin glass wall can be put at the exit of a capillary. Hence, we can irradiate the focused beam to samples which can not be set in vacuum like living human cells [5]. In addition, this technique allows us to construct the focusing system small with low cost. The density of the ion beam flux can be increased by 10-1000 times by using the tapered glass capillary which has smaller inner diameter at the exit than that at the entrance. This effect is called as "focusing effect". On the other hand, the ion beam can be deflected by tilting the capillary, which is called as "guiding effect". Both effects are considered due to reflection (or scattering) of the beam on the capillary inner wall [2 - 4].

The capillary technique might be useful especially when we apply slow positrons to the biological/medical science field [6], because it has a potential to inject positrons into locally selected area of living cells. In this paper, we report experimental results for confirming guiding effect of slow positrons using a non-tapered capillary as a first step of the importing of capillary techniques to the slow positron beam.

\section{Experimental Setup}

Figure 1 shows the experimental setup around a capillary which is connected to the AIST-positron beamline [1]. Leakage magnetic field around the capillary from the upstream magnetic transport system was $0.4 \mathrm{mT}$. Positron beam intensity was a few $10^{4} \mathrm{e}^{+} / \mathrm{s}$ after the tungsten collimator $(10 \mathrm{~mm}$ in the thickness and $2 \mathrm{~mm}$ in inner diameter). Beam energy was $10 \mathrm{keV}$. The glass capillary was 54 $\mathrm{mm}$ in length and $3 \mathrm{~mm} / 1.8 \mathrm{~mm}$ in outer/inner diameter. It was mounted on an $x y \theta \phi$-movable stage. The rotation center for $\theta / \phi$ motion was set at the center of the capillary entrance. Micro channel plates 
(MCPs: effective diameter $10 \mathrm{~mm}$ ) with a phosphor screen was mounted $80 \mathrm{~mm}$ downstream from the collimator. A charge coupled device (CCD) camera was used to record the images obtained by the MCP/phosphor screen. Spatial resolution of the image recorded with this system was $\approx$ $190 \mu \mathrm{m}$. Front bias of the MCPs was set to the same value of the surrounding potential $\left(V_{m}=0 \mathrm{~V}\right)$ in order to prevent disturbing the positron orbit by an electric field. The vacuum pressure around capillary was $1 \times 10^{-5} \mathrm{~Pa}$. Exposing the $\mathrm{MCP} /$ phosphor to the collimated beam directly, i.e. without the capillary, it was confirmed that the divergence of the direct beam was $\approx 3$ mrad which was much smaller than the aspect ratio of the capillary $\alpha=1.8 / 54 \approx 33 \mathrm{mrad}$.

The experimental procedure was as follows. Firstly, the axis of the capillary was adjusted to the beam axis, and the position was defined as the origin, $(\mathrm{x}, \mathrm{y}, \theta, \phi)=(0,0,0,0)$. Then, keeping $\mathrm{x}=\mathrm{y}=\phi=0$, the $\mathrm{MCP} /$ phosphor images were recorded as a function of $\theta$.

\section{Results and discussion}

Experimental results for $\theta=0.0,-26.2$ and $-43.6 \mathrm{mrad}$ are shown in Fig. 2 (a). The exposure time of the CCD camera for each image was $30 \mathrm{~s}$. The beam spot moved in the direction of $\mathrm{x}$ with $\theta$ as it follows the position of the capillary exit. These images were not changed even if $V_{m}=-150 \mathrm{~V}$, which confirms that these images are not affected by stray secondary electrons. Influence of positronium in this experiment is negligible because its formation cross section is negligibly small [7].

Figure 2(b) shows the intensity profile of the images projected to the $\mathrm{x}$ axis in comparison with that of the beam without capillary. The beam transported through the capillary appeared around $\mathrm{x}=-3$ when $\theta=-43.6 \mathrm{mrad}$, while the beam without capillary did not spread there. This fact is an evidence that the glass capillary has the guiding effect for the $10 \mathrm{keV}$ positron beam.
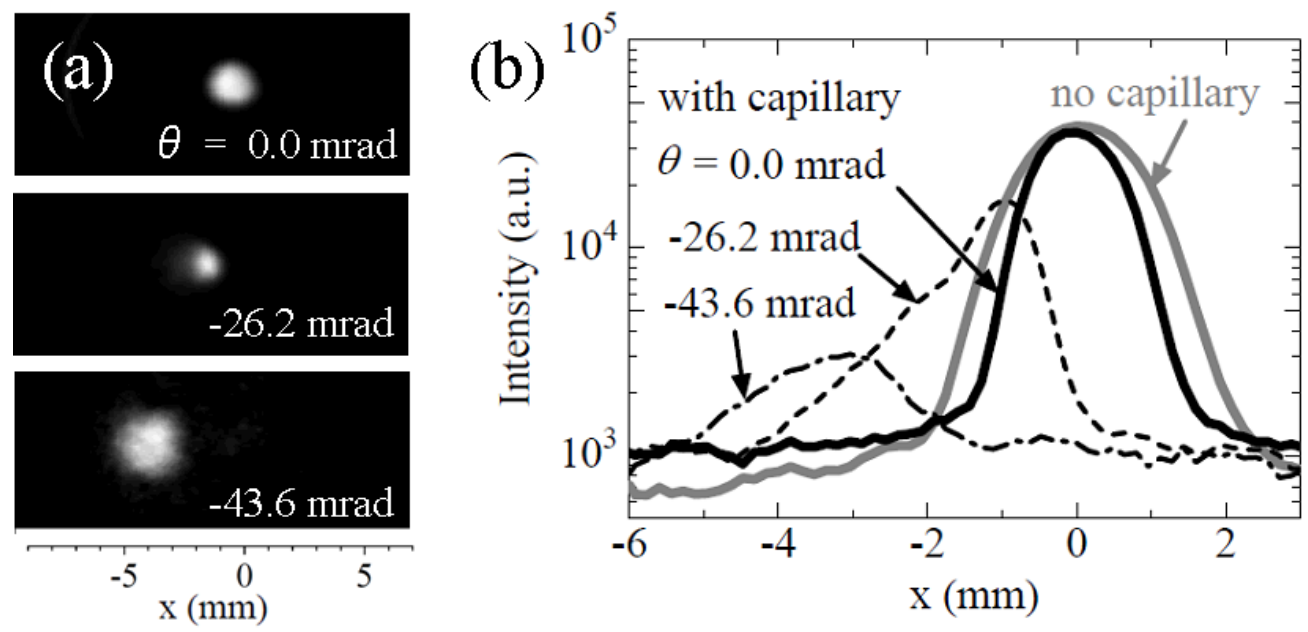

Fig. 2 (a) MCP /phosphor iamges recorded by the CCD camera and (b) beam intensity profile projected to the $\mathrm{x}$ axis of the images obtained with and without capillary.

Figure 3(a) shows the relation between the peak position on the $\mathrm{x}$ axis of the intensity profile and $\theta$. Gradient $(\mathrm{dx} / \mathrm{d} \theta)$ was changed at $|\theta| \approx \alpha$. These results are explained as follows. In case of $|\theta|<\alpha$, a direct beam can reach the MCPs (see Fig. 3(c)) and its peak center is geometrically given by $\mathrm{x} \approx$ $(54 / 2) \theta$. On the other hand, in case of $|\theta|>\alpha$, only the positrons reflected (scattered) toward the exit in the capillary can arrive at MCP and its peak position is geometrically given by $\mathrm{x} \approx 78 \theta$. (The length between the entrance of capillary and the MCP is $78 \mathrm{~mm}$.) These are in good agreement with the 
experimental results (see Fig. 3(a)). Figure 3(b) shows $\theta$ dependence of integrated intensity deduced from each image. The $\mathrm{MCP} /$ phosphor photo-intensity for $\theta=-43.6 \mathrm{mrad}$ was about $1 / 10$ compared with that for $\theta=0$. Energy distribution of the positrons transported through the capillary was not measured. Since the quantum efficiency $\varepsilon$ of the MCP depends on the beam energy, it is difficult to deduce the accurate beam intensity from the photo-intensity. However, $\varepsilon$ for electrons changes slowly in the considering energy region, for example $\varepsilon$ around $10 \mathrm{keV}$ is about $30 \%$ lower than that around 5 $\mathrm{keV}$ [8]. This implies that Fig. 3(b) reflects the $\theta$ dependence of beam intensity passed through the capillary. Further investigation with counting experiment is necessary for quantitative evaluation of
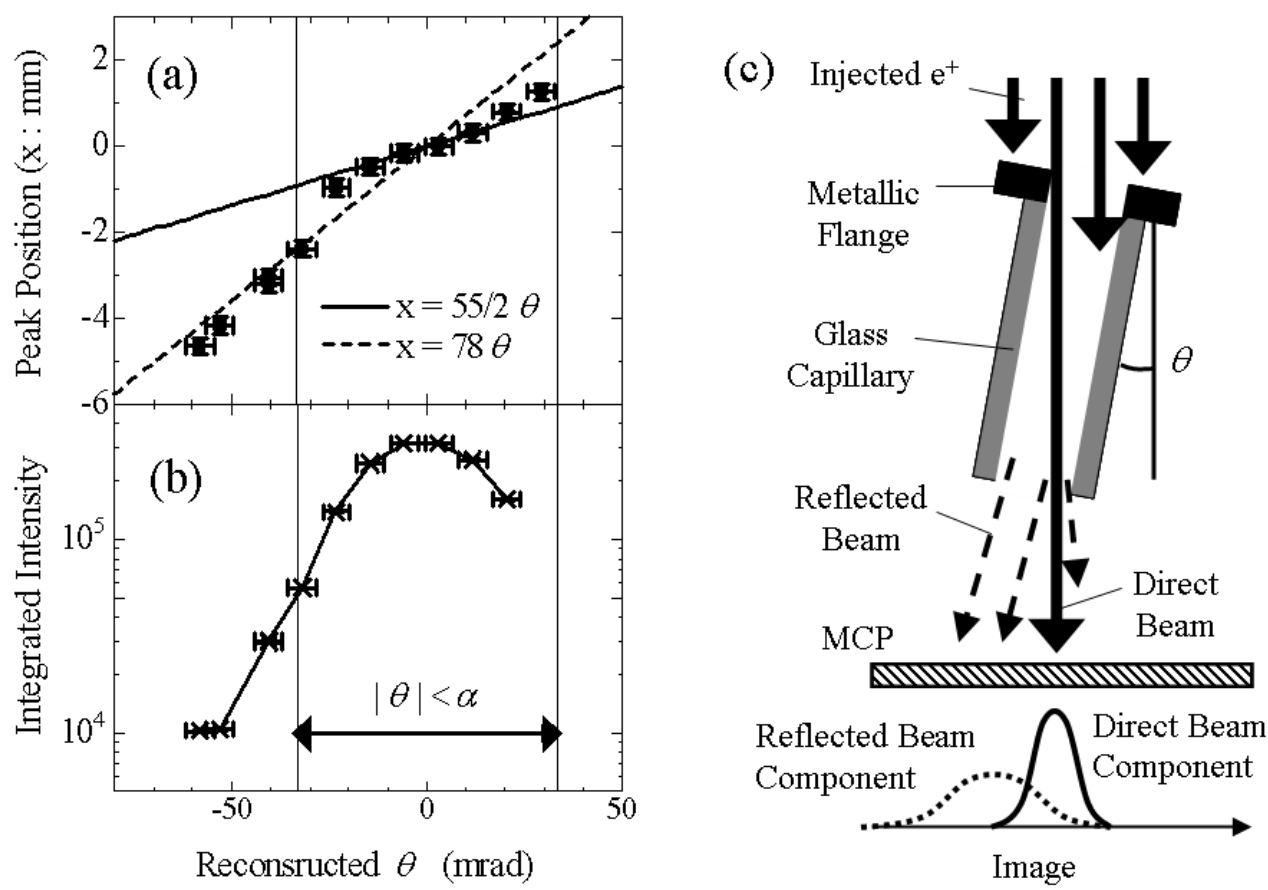
the beam intensity.

Fig. 3 (a) The peak position on the $\mathrm{x}$ axis of the image intensity profile. (b) The integrated intensity of images as a function of $\theta$. (c) Only reflected (scattered) positrons in the capillary can arrive at MCP in case of $|\theta|>\alpha$, while a direct beam can also reach the MCPs in case of $|\theta|<\alpha$.

\section{Summary}

We observed guiding of positron beam with a glass capillary. This implies the possibility of "focusing effect" for positron beam with suitable tapered capillaries. Further investigation of dependences of the guiding and focusing effects on capillary shape, capillary materials, beam parameters, charge up due to positron irradiation $[3,9]$ are under planning.

\section{References}

[1] N. Oshima et al., J. Appl. Phys. Vol. 103 (2008), p. 094916

[2] T. Nebiki et al., J. Vac. Sci. Technol. A Vol. 21 (2003), p. 1671

[3] T. Ikeda et al., Appl. Phys. Lett. Vol. 89 (2006), p. 163502

[4] T. M. Kojima et al., J. Phys. Soc. Jpn. Vol. 76 (2007), p. 093501

[5] Y. Iwai et al., Appl. Phys. Lett. Vol. 92 (2008), p. 023509

[6] G. Liu et al., Phys. Stat. Sol. (c) Vol .4 (2007), p. 3912

[7] G. Laricchia, in: Positron Spectroscopy of Solids, edited by A. Dupasquier and A. P. Mills jr., (1993), p. 401

[8] MCP assembly technical documents by Hamamatsu Photonics, in Japanese.

[9] P. G. Coleman, S. Kuna, and R. Grynszpan, Mater. Sci. Forum. Vol. 225-257 (1997), p. 668 